Noname manuscript No.

(will be inserted by the editor)

Klaus Morawetz

\title{
Asymmetric Bethe-Salpeter equation for pairing and condensation
}

Received: date / Accepted: date

\begin{abstract}
The Martin-Schwinger hierarchy of correlations are reexamined and the three-particle correlations are investigated under various partial summations. Besides the known approximations of screened, ladder and maximally crossed diagrams the pair-pair correlations are considered. It is shown that the recently proposed asymmetric Bethe-Salpeter equation to avoid unphysical repeated collisions is derived as a result of the hierarchical dependencies of correlations. Exceeding the parquet approximation we show that an asymmetry appears in the selfconsistent propagators. This form is superior over the symmetric selfconsistent one since it provides the Nambu-Gorkov equations and gap equation for fermions and the Beliaev equations for bosons while from the symmetric form no gap equation results. The selfenergy diagrams which account for the subtraction of unphysical repeated collisions are derived from the pair-pair correlation in the three-particle Green's function. It is suggested to distinguish between two types of selfconsistency, the channel-dressed propagators and the completely dressed propagators, with the help of which the asymmetric expansion completes the Ward identity and is $\Phi$-derivable.
\end{abstract}

K. Morawetz

Münster University of Applied Science, Stegerwaldstrasse 39, 48565 Steinfurt, Germany

International Institute of Physics (IIP) Federal University of Rio Grande do Norte Av. Odilon Gomes de Lima 1722, 59078-400 Natal, Brazil

Max-Planck-Institute for the Physics of Complex Systems, 01187 Dresden, Germany

Tel.: +49 2551962411

Fax: +492551962811

E-mail: morawetz@fh-muenster.de 
Keywords Many-body theory · Correlations in Nonequilibrium · BetheSalpeter equation

PACS 71.10.-w $\cdot 05.30 .-\mathrm{d} \cdot 74.20 .-\mathrm{z} \cdot 05.60 . \mathrm{Gg} \cdot 67.85 . \mathrm{d} \cdot 82.20 . \mathrm{-w}$

\section{Introduction}

Though pairing and condensation phenomena like superconductivity belong to the most exciting and long studied effects, their many-body theoretical treatments lacks often a systematic justifications. For example a particlenumber non-conserving assumption is often accepted by anomalous propagators in order to obtain the desired structure of Green's functions. Systematic many-body approximations like the ladder T-matrix [1,2,3,4,5, leading to the Bethe-Salpeter equation had to face the puzzle that the selfconsistent propagators describing the many-body medium have to be assumed in asymmetric form in order to obtain the gap equation. This was first observed by Kadanoff and Martin [1 and this approximation has been used mainly in the theory of superconductivity [4,5,6]. For an overview about different T-matrix approximations see 7 . Though any T-matrix based on the Bethe-Salpeter equation becomes unstable at the critical temperature 3 where the T-matrix diverges, the corresponding selfenergy fails to describe the superconducting gap [8] if we do not assume an asymmetry in the internal propagators.

Recently a way has been proposed to obtain such asymmetry by demanding that particles should not interact with the same state repeatedly $[9]$. Such non-physical repetition leads to an incorrect description of the coherent part of the scattering [10, and an incorrect single-particle Green function $G$. These non-physical repeated collisions destroy the superconducting gap in the energy spectrum of $G$. Using the Soven scheme 11 the repeated collisions can be avoided 9 and the gap in the superconducting state can be described as well as the normal state. This subtraction scheme can be written in a form equivalent to the anomalous propagator approach such that these propagators appear as consequence of the theory and need not to be assumed ad-hoc [12. Though in this way a physical understanding of a deficiency in selfconsistent many-body expansion has already been achieved, it is desirable to see how a systematic expansion of correlations can account for such corrections.

The necessity to introduce asymmetric propagators can be seen from another physical system of dense correlated plasmas. There the motion of ions are lowered by the dynamics of the screening cloud since a deceleration force appears which is caused by the deformed screening cloud surrounding the charge. This effect known as Debye-Onsager relaxation effect has been devoted much interest 13, 14, 15, 16, 17, 18, 19, 20, which was first derived within the theory of electrolytes [21,22,23,24,25]. It turned out that only an asymmetric assumption [20] about the mutual screening can correctly describe the Onsager result [16, 17, 19.

Due to the widespread usage of T-matrix approximations ranging from nuclear physics for both equilibrium [26, 27, 28, and non-equilibrium [29, 30] problems, to the theory of moderately dense gases [31] and liquid ${ }^{3} \mathrm{He}$ [32, as well as to electron-electron correlations in molecules and solids $33,34,35$, 
36. 37 it is desirable to clarify the correct structure of the Bethe-Salpeter equation and to specify the conditions under which the corresponding approximate forms can be used.

Here we will present a derivation of modified Bethe-Salpeter equations from first-principles which shows indeed that the hierarchical structure of correlation functions leads to such asymmetric selfconsistency as necessary to describe pairing. We will present the derivation in terms of the causal n-particle Green's functions. The cummulant expansion yields a hierarchy of correlation functions which leads already to the asymmetric result assumed by Kadanoff and Martin in binary correlation approximation.

We will then proceed and investigate the three-particle correlation and will select the processes between two pairs of particles which allow to consider the repeated collisions. We will show that the systematic cummulant expansion leads to the correct subtraction in the channels with condensates called singular channels. The idea of derivation follows here the centennial overview of many-body approximations by Heinz Puff [38] which is suited for nonequilibrium Green's function expansions. Therefore all outlined formalism holds in nonequilibrium as well as equilibrium. The expansion scheme of three-particle correlations will lead to the appearance of two different kinds of selfconsistent propagators in each considered channel. These will be the channel-dressed and the completely dressed propagators.

The outline of the paper is as follows. In the next chapter a scheme of Green's functions with respect to the cummulant expansion is shortly presented. This is performed with the help of the variation technique including an external potential in order to ensure the Ward-identity, $\phi$-derivability and conservation laws which will be shown in the appendix. Then we consider in the third chapter the binary approximation neglecting any three-particle correlation. This will lead to the Kadanoff- and Martin approximation providing the correct gap equation. We discuss then the repeated collisions and how it can be avoided by a proper subtraction scheme. Though leading to the same gap equation the underlying approximation is beyond the KadanoffMartin form. To justify this subtraction scheme we consider in chapter IV the three-particle correlations. We demonstrate that the expansion of threeparticle correlations in different channels leads to the distinction of two kinds of selfconsistent propagators, the channel dressed and the completely dressed one. Then we focus on the correlations between two pairs. This provides a special contribution from the pairing or condensation channel to the different channels contained in the three-particle correlations. It turned out that this selfenergy contribution provides exactly the subtracted scheme discussed in chapter III and which was proposed in the literature. In the appendix we proof the Ward identity and the $\Phi$-derivability in order to ensure conservation laws. We summarize and emphasize that the hierarchical dependence of correlation care for the avoidance of unphysical repeated collisions itself. As a consequence the T-matrix schemes used so far should be revised as soon as singular channels appear like in phenomena of condensation or pairing in such a way that an asymmetry in the selfconsistency is proposed. 


\section{Decoupling scheme of correlations}

The n-particle causal Green's function

$$
\begin{aligned}
G\left(1,2, \ldots, n, 1^{\prime}, 2^{\prime}, \ldots, n^{\prime} ; U\right) & =\frac{1}{i^{n}} \frac{\left\langle T S \Psi_{1} \ldots \Psi_{n} \Psi_{1^{\prime}}^{+} \ldots \Psi_{n^{\prime}}^{+}\right\rangle}{\langle T S\rangle} \\
S & =\mathrm{e}^{\int d 1 \Psi_{1}^{+} \Psi_{1} U(1)}
\end{aligned}
$$

of a system under the influence of an external field $U(1)$, where numbers $1,2, \ldots$ sign cumulative indices like space, time, $\ldots$ coordinates, can be formally represented by a generating functional

$$
G\left(1,2, \ldots, n, 1^{\prime}, 2^{\prime}, \ldots ., n^{\prime} ; U\right)=\left.\partial_{\eta_{n^{\prime}}} \ldots \partial_{\eta_{1^{\prime}}} \partial_{\lambda_{1}} \ldots \partial_{\lambda_{n}} \mathcal{G}[\lambda, \eta]\right|_{\lambda=\eta=0}
$$

with

$$
\mathcal{G}[\lambda, \eta]=1+\sum_{1}^{n} \frac{1}{(n !)^{2}} \int d 1 \ldots d n d 1^{\prime} \ldots d n^{\prime} \lambda_{n} \ldots \lambda_{1} G\left(1 \ldots n^{\prime} ; U\right) \eta_{1^{\prime}} \ldots \eta_{n^{\prime}}
$$

where $\eta, \lambda$ are Bose/Fermi-commuting auxiliary fields.

The Martin-Schwinger hierarchy [39] coupling the one-particle Green's function to the two-particle one,

$$
\left[i \partial_{t_{1}}+\frac{\nabla_{r_{1}}^{2}}{2 m}-U(1)\right] G\left(11^{\prime} ; U\right)=\delta\left(1-1^{\prime}\right) \mp i \int d \overline{1} V(1, \overline{1}) G\left(1 \overline{1} 1^{\prime} \overline{1}^{+} ; U\right),
$$

is expressed in terms of this generating functional by

$$
\left[i \partial_{t_{1}}+\frac{\nabla_{r_{1}}^{2}}{2 m}-U(1)\right] \partial_{\lambda_{1}} \mathcal{G}=\eta_{1} \mathcal{G} \mp i \int d \overline{1} V(1, \overline{1}) \partial_{\eta_{\overline{1}+}} \partial_{\lambda_{\overline{1}}} \partial_{\lambda_{1}} \mathcal{G} .
$$

The upper sign denotes fermions the lower bosons hereafter. It is now useful to introduce the correlated n-particle Green's function as the cummulant expansion [40] due to a new generating functional $\mathcal{G}_{c}$,

$$
\mathcal{G}[\lambda, \eta]=\exp ^{\mathcal{G}_{c}[\lambda, \eta]}
$$

with

$$
\mathcal{G}_{c}[\lambda, \eta]=\sum_{1}^{n} \frac{1}{(n !)^{2}} \int d 1 \ldots d n d 1^{\prime} \ldots d n^{\prime} \lambda_{n} \ldots \lambda_{1} G_{c}\left(1 \ldots n^{\prime} ; U\right) \eta_{1^{\prime}} \ldots \eta_{n^{\prime}} .
$$

The comparison of (6), (7) and (3) with respect to the orders of $\lambda, \eta$ reveals the cummulant expansion. The first order reads

$$
G\left(11^{\prime} ; U\right)=G_{c}\left(11^{\prime} ; U\right),
$$


i.e. the single-particle Green's function equals its correlated part. The second order shows just the separation of the Hartree-Fock term from the twoparticle Green's function

$$
\begin{aligned}
& G\left(121^{\prime} 2^{\prime} ; U\right)=G_{c}\left(121^{\prime} 2^{\prime} ; U\right) \\
& +G\left(11^{\prime} ; U\right) G\left(22^{\prime} ; U\right) \mp G\left(12^{\prime} ; U\right) G\left(21^{\prime} ; U\right) .
\end{aligned}
$$

The third order is given by all possible exchanges of three one-particle Green's functions together with all possible exchanges of the two-particle correlated Green's function and a one-particle Green's function.

Introducing (6) into (5) and notating the inverse Hartree-Fock Green's function with

$$
\begin{aligned}
& G_{\mathrm{HF}}^{-1}(12 ; U)=-i V(12) G(12 ; U) \\
& +\left[i \partial_{t_{1}}+\frac{\nabla_{r_{1}}^{2}}{2 m}-U(1) \pm i \int d \overline{1} V(1 \overline{1}) G\left(\overline{1} \overline{1}^{+} ; U\right)\right] \delta(1-2)
\end{aligned}
$$

one can invert the differential equation (5) into an integral equation

$$
\partial_{\lambda_{1}} \mathcal{G}_{c}=\mathcal{I}(1)
$$

with

$$
\begin{aligned}
& \mathcal{I}(1)=\int d \overline{1} G_{\mathrm{HF}}(1 \overline{1} ; U) \eta_{\overline{1}} \mp i \int d \overline{1} d \overline{2} G_{\mathrm{HF}}(1 \overline{1} ; U) V(\overline{1} \overline{2}) \\
& \times\left\{\partial_{\eta_{\overline{2}+}} \partial_{\lambda_{\overline{2}}} \partial_{\lambda_{\overline{1}}} \mathcal{G}_{c}\right. \\
& +\left[\partial_{\eta_{\overline{2}}+} \partial_{\lambda_{\overline{2}}} \mathcal{G}_{c}-G\left(\overline{2} \overline{2}^{+} ; U\right)\right] \partial_{\lambda_{\overline{1}}} \mathcal{G}_{c} \\
& \mp\left[\partial_{\eta_{\overline{2}}+} \partial_{\lambda_{\overline{1}}} \mathcal{G}_{c}-G\left(\overline{1} \overline{2}^{+} ; U\right)\right] \partial_{\lambda_{\overline{2}}} \mathcal{G}_{c} \\
& \left.\partial_{\eta_{\overline{2}+}} \mathcal{G}_{c}\left[\partial_{\lambda_{\overline{2}}} \partial_{\lambda_{\overline{1}}} \mathcal{G}_{c}+\partial_{\lambda_{\overline{2}}} \mathcal{G}_{c} \partial_{\lambda_{\overline{1}}} \mathcal{G}_{c}\right]\right\} .
\end{aligned}
$$

Using (7) we see that from the definition the correlated Green's functions can be represented by functional derivatives of (11),

$$
\begin{aligned}
G_{c}\left(11^{\prime} ; U\right) & =\left.\partial_{\eta_{1^{\prime}}} \partial_{\lambda_{1}} \mathcal{G}_{c}\right|_{\lambda=\eta=0} \\
& =\left.\partial_{\eta_{1^{\prime}}} \mathcal{I}(1)\right|_{\lambda=\eta=0} \\
G_{c}\left(121^{\prime} 2^{\prime} ; U\right) & =\left.\partial_{\eta_{2^{\prime}}} \partial_{\eta_{1^{\prime}}} \partial_{\lambda_{1}} \partial_{\lambda_{2}} \mathcal{G}_{c}\right|_{\lambda=\eta=0} \\
& =\left.\mp \partial_{\eta_{2^{\prime}}} \partial_{\eta_{1^{\prime}}} \partial_{\lambda_{2}} \mathcal{I}(1)\right|_{\lambda=\eta=0} \\
G_{c}\left(1231^{\prime} 2^{\prime} 3^{\prime} ; U\right) & =\left.\partial_{\eta_{3^{\prime}}} \partial_{\eta_{2^{\prime}}} \partial_{\eta_{1^{\prime}}} \partial_{\lambda_{1}} \partial_{\lambda_{2}} \partial_{\lambda_{3}} \mathcal{G}_{c}\right|_{\lambda=\eta=0} \\
& =\left.\partial_{\eta_{3^{\prime}}} \partial_{\eta_{2^{\prime}}} \partial_{\eta_{1^{\prime}}} \partial_{\lambda_{2}} \partial_{\lambda_{3}} \mathcal{I}(1)\right|_{\lambda=\eta=0}
\end{aligned}
$$

and so on. We drop the notation of the explicit dependence on the external potential $\mathrm{U}$ in the following. The first equation of (13) yields for the oneparticle correlated Green's function

$$
G_{c}\left(11^{\prime}\right)=G_{\mathrm{HF}}\left(11^{\prime}\right) \mp i \int d \overline{1} \overline{2} G_{\mathrm{HF}}(1 \overline{1}) V(\overline{1}, \overline{2}) G_{c}\left(\overline{1} \overline{2} 1^{\prime} \overline{2}^{+}\right)
$$


and is nothing else but the Martin-Schwinger hierarchy (44) written for the correlated parts and in integral form.

The integral equation for the two-particle Green's function is more involved and reads from the second equation of (13)

$$
\begin{aligned}
& G_{c}\left(121^{\prime} 2^{\prime}\right)=i \int d \overline{1} d \overline{2} G_{\mathrm{HF}}(1 \overline{1}) V(\overline{1} \overline{2})\left\{\mp G_{c}\left(\overline{2} \overline{1} 22^{+} 1^{\prime} 2^{\prime}\right)\right. \\
& +G\left(2 \overline{2}^{+}\right)\left[G_{c}\left(\overline{1} \overline{2} 1^{\prime} 2^{\prime}\right)+G\left(\overline{2} 2^{\prime}\right) G\left(\overline{1} 1^{\prime}\right) \mp G\left(\overline{2} 1^{\prime}\right) G\left(\overline{1} 2^{\prime}\right)\right] \\
& +G_{c}\left(\overline{1} 2 \overline{2}^{+} 2^{\prime}\right) G\left(\overline{2} 1^{\prime}\right)+G_{c}\left(\overline{1} 1^{\prime} 2 \overline{2}^{+}\right) G\left(\overline{2} 2^{\prime}\right) \\
& \left.\mp G_{c}\left(\overline{2} 2 \overline{2}^{+} 2^{\prime}\right) G\left(\overline{1} 1^{\prime}\right) \mp G_{c}\left(\overline{2} 21^{\prime} \overline{2}^{+}\right) G\left(\overline{1} 2^{\prime}\right)\right\} .
\end{aligned}
$$

This equation is graphical presented in figure 1 and is exact so far. Since the following algebra is somewhat involved it is easier to perform it in terms of diagrammatic presentation. Therefore we design a complete selfconsistent one-particle Green's function with a thick full arrow, the interaction with a broken line and the two-, three-, and four-particle Green's functions with a square containing two, three and four legs. The Hartree-Fock Green's function is designed as thin arrow. Corresponding upper signs denote fermions and lower signs bosons. The numbering of in and outgoing channels are in the direction from bottom to top. Lines without an arrow indicates ends of twoand three particle Green's functions which are not connected with a oneparticle Green's function.

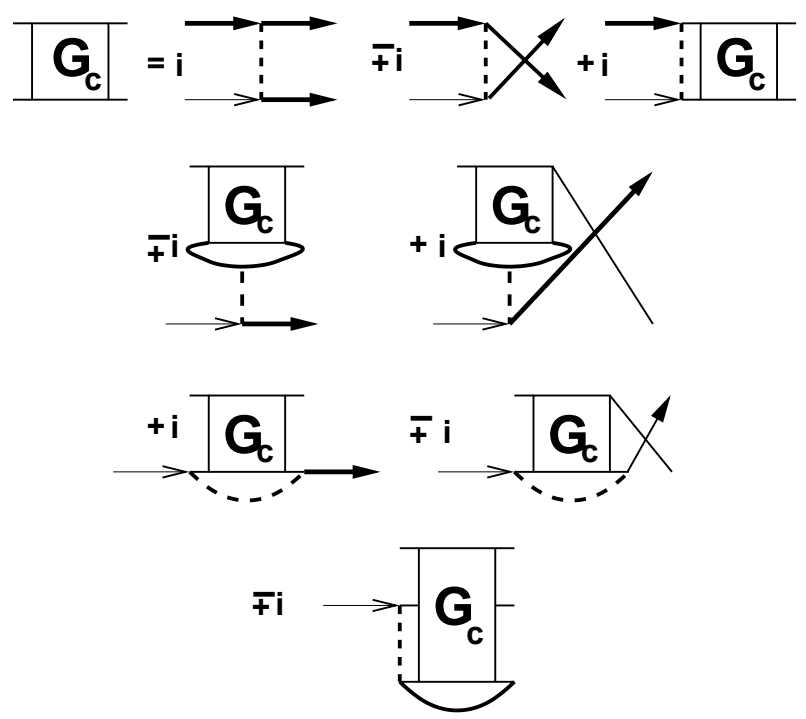

Fig. 1 The equation for the correlated two-particle Green's function. The thin open arrows are Hartree-Fock Green's functions, the thick arrows are full Green's functions. The first line represents the ladder approximation and the second and third line exchange channels. 
From the derivation above it is not hard to see that the property

$$
G_{c}\left(121^{\prime} 2^{\prime} ; U\right)=\mp G_{c}\left(211^{\prime} 2^{\prime} ; U\right)=\mp G_{c}\left(122^{\prime} 1^{\prime} ; U\right)
$$

is ensured. In other words the criterion $(\mathrm{A})$ and $(\mathrm{B})$ of Kadanoff and Baym is fulfilled and we have a conserving approximation, i.e. the energy, momentum and density is conserved [41. Please note that the corresponding diagrams in the second and third line in figure 1 cancel each other for fermions and contact interaction, i.e. they become small for short-range interactions.

\section{Two-particle correlations}

\subsection{Binary collision approximation}

As a first very drastic approximation we want to consider the binary correlation approximation which consists in neglecting the correlated three-particle Green's function in (15), i.e. the last diagram in figure (11). Further we neglect also the second and third line since they vanish for fermions and shortrange interaction. This results into the known ladder approximation or BetheSalpeter equation. Amazingly we have now obtained that the intermediate propagators of the Bethe-Salpeter equation have to be considered asymmetrically. Indeed, the vertex

$$
\begin{aligned}
G_{c}\left(121^{\prime} 2^{\prime}\right)= & \int d \overline{1} d \overline{2} d \overline{1}^{\prime} d \overline{2}^{\prime} G_{\mathrm{HF}}(1 \overline{1}) G(2 \overline{2}) \Gamma\left(\overline{1} \overline{2} \overline{1}^{\prime} \overline{2}^{\prime}\right) \\
& \left.\times G\left(\overline{1}^{\prime} 1^{\prime}\right) G\left(\overline{2}^{\prime} 2^{\prime}\right)\right)
\end{aligned}
$$

can be represented in symmetrized form

$$
\Gamma\left(121^{\prime} 2^{\prime}\right)=T\left(121^{\prime} 2^{\prime}\right) \mp T\left(122^{\prime} 1^{\prime}\right)
$$

by the T-matrix

$$
\begin{aligned}
& T\left(121^{\prime} 2^{\prime}\right)=i V(12) \delta\left(1-1^{\prime}\right) \delta\left(2-2^{\prime}\right) \\
& +i \int d \overline{1} d \overline{2} V(12) G(2 \overline{2}) G_{\mathrm{HF}}(1 \overline{1}) T\left(\overline{1} \overline{2} 1^{\prime} 2^{\prime}\right) .
\end{aligned}
$$

The asymmetry appears such that a full Green function $G_{c}$ is combined with a Hartree-Fock Green's function. This asymmetry is maintained if we go systematically to higher-order approximations neglecting 4-particle correlated Green's functions as we will see in the next chapter. It is a result of the hierarchical structure of the equations of motion. If one compares with the linearized parquet approximations [42,43], the Hartree-Fock propagator $G_{\mathrm{HF}}$ in figure (11) is replaced by the full one $G_{c}$ which includes nonphysical processes. We will see in the next chapter that the result derived here by the hierarchy avoids such non-physically multiple scatterings with the same channel. Therefore we consider the asymmetric form in figure 1 as superior to the parquet approximation. 


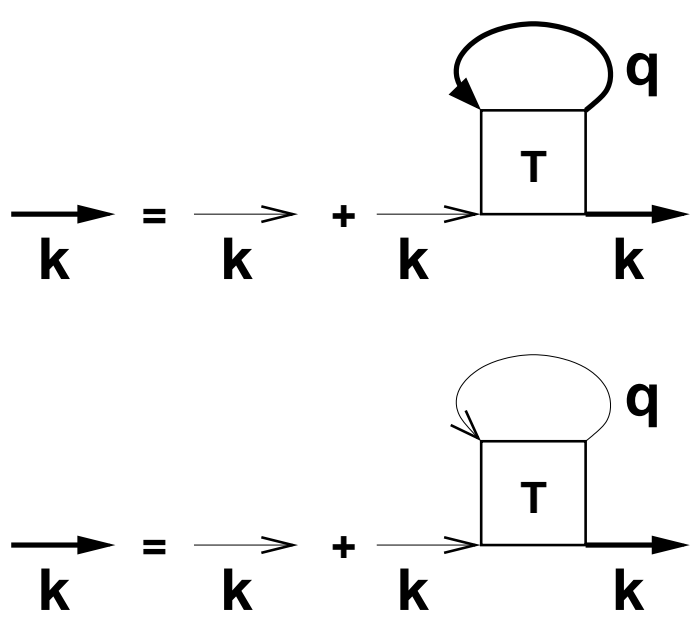

Fig. 2 The Dyson equation in ladder approximation for the symmetric selfconsistency (above) and the Kadanoff-Martin asymmetric one derived here (below).

In fact this asymmetry has been recognized as being necessary to obtain the gap equation for pairing [44. It was first observed by Kadanoff and Martin [1] and used later on [4,5,9,45] as an ad-hoc approximation seemingly violating the symmetry of equations and consequently violating conservation laws. This has remained puzzling since a worse approximation leads obviously to better results. Recently it turned out that the repeated collisions 9 with the same particle are responsible for this artifact.

\subsection{Derivation of Gap equation}

Let us illustrate this by comparing the Dyson equation with symmetric and with asymmetric selfconsistency in figure 2. The T-matrix has poles at bound states and at the pairing and becomes separable near these poles, $T=\mp \triangle \triangle$ where the upper sign is for fermions and the lower for bosons. The two different Dyson equations for the propagator with respect to the symmetry of selfconsistency is illustrated in the next figure 3 . The first symmetrical

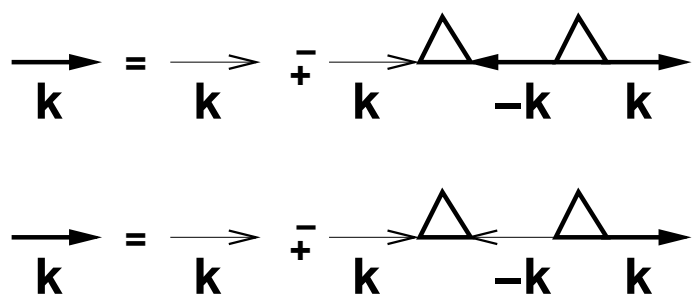

Fig. 3 The propagator equations resulting from figure 2 selfconsistently (above), Kadanoff-Martin asymmetric (below). The upper/lower sign stands for Fermi/Bose systems. 
selfconsistent Dyson equation leads to the propagator

$$
G(\omega, \mathbf{k})=\frac{1}{\omega-\epsilon_{\mathbf{k}} \pm \Delta^{2} G(-\omega,-\mathbf{k})}
$$

which shows no pole and no gap equation. Considering the second asymmetrical Kadanoff-Martin approximation of figure 3 we obtain

$$
G(\omega, \mathbf{k})=\frac{1}{\omega-\epsilon_{\mathbf{k}} \mp \frac{\Delta^{2}}{\omega+\epsilon_{-\mathbf{k}}}}
$$

which possesses the typical two-pole structure of the BCS gap equation in the case of fermions. Therefore the Kadanoff and Martin approximation is superior to the symmetric selfconsistent ladder approximation and appears as a consequence of the hierarchical dependencies of correlations as derived above.

What has this now to do with avoiding repeated collisions? Repeated collisions of two particles in the same state are unphysical since the particles move apart from each other after the collision. Therefore we have to ensure that due to selfconsistency such collisions with the same state do not appear. In fact as can be seen in figure 4, the selfconsisent Dyson equation does not ensure that the momentum of the repeated collisions, $\mathbf{p}$, is unequal to the momentum of the incoming particle $\mathbf{k}$. If this would be the case the particle will scatter with a particle in the same state again. These repeated collisions have to be removed from the T-matrix and the correct gap equation appears and the condensate can be described without asymmetrical ad-hoc assumptions about selfconsistency. The advantage of eliminating only the contributions of single channels as proposed in Refs. 9] and [45] is that the formation of pairs and their condensation can be described within the same approximation. Explicitly, we split the selfenergy into different channels, $\Sigma=$

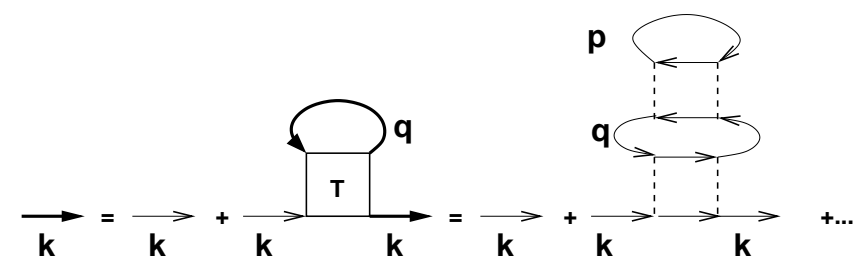

Fig. 4 The iteration of the selfconsistent Dyson equation of figure 2 leading to repeated collision with momentum $\mathbf{p}, \mathbf{q}$.

$\sum_{j} \Sigma_{j}$ where we assume the condensation or pairing to appear in the channel $i$ called the singular channel. Now we define a subtracted propagator

$$
G_{\chi}=G-G_{\chi} \Sigma_{i} G
$$

where the T-matrix of the channel carrying the pole, $T_{i}$, is closed by the subtracted propagator $\Sigma_{i}=T_{i} \bar{G}_{\hat{\chi}}$. Writing the Dyson equation explicitly 
leads to the full propagator in momentum-energy representation $p=(\omega, \mathbf{p})$ 12

$$
G(p)=\frac{\omega+\epsilon+\bar{\Sigma}_{11}}{\left(\omega+\epsilon+\bar{\Sigma}_{11}\right)\left(\omega-\epsilon-\Sigma_{11}\right) \mp \Sigma_{12}^{2}}
$$

where $\Sigma_{11}(p) \equiv \Sigma(p)-\Sigma_{i}(p)$ and $\Sigma_{12}(p) \equiv \Delta(p)$ and the upper/lower sign stands for fermions/bosons. The expression (23) is nothing else but the Beliaev form for bosons [46] or the Nambu-Gorkov form for fermions [47] and can be written also in matrix notation with off-diagonal elements [12.

Though we have shown above that the Kadanoff-Martin approximation leads already to a two-pole Green's function (21) necessary for the gap equation, the alert reader has noticed that the subtraction of unphysical multiple scattering events has assumed a different intermediate propagator than the Kadanoff-Martin approximation though leading to the same gap propagator (23). The Kadanoff-Martin approximation requires to use the Hartree-Fock propagator as derived above by approximating the hierarchy of correlations at the binary level. For the subtraction of repeated collisions we have used instead the propagator written with the help of (22)

$$
G_{\chi}=G_{\mathrm{HF}}+G_{\mathrm{HF}}\left(\Sigma-\Sigma_{\mathrm{HF}}-\Sigma_{i}\right) G_{\chi}
$$

which shows that the subtracted propagator is beyond the Hartree-Fock one. How can we derive such a form from the hierarchy of correlations?

\section{Three-particle approximations}

\subsection{Generic structure}

For this purpose we go one step further and approximate the equation for the three-particle correlation represented by the last line of (13). It shows the coupling to the four-particle Green's function and is quite lengthy. For the sake of legibility we abbreviate the interchanges of indices of the corresponding foregoing expressions by denoting them in the following formula within the same kind of brackets,

$$
\begin{aligned}
& G_{c}\left(1231^{\prime} 2^{\prime} 3^{\prime}\right)=\mp i \int d \overline{1} d \overline{2} G_{\mathrm{HF}}(1 \overline{1}) V(\overline{1} \overline{2})\left\{G_{c}\left(23 \overline{2} \overline{1} \overline{2}^{+} 1^{\prime} 2^{\prime} 3^{\prime}\right)\right. \\
& \mp G_{c}\left(2 \overline{2} \overline{1} 1^{\prime} 2^{\prime} 3^{\prime}\right) G_{c}\left(3 \overline{2}^{+}\right) \mp(2 \leftrightarrow 3) \\
& +\left\{G_{c}\left(23 \overline{2} \overline{2}^{+} 2^{\prime} 3^{\prime}\right) G_{c}\left(\overline{1} 1^{\prime}\right) \mp(\overline{1} \leftrightarrow \overline{2})\right. \\
& \mp G_{c}\left(2 \overline{2} \overline{2}^{+} 3^{\prime}\right) G_{c}\left(3 \overline{1} 1^{\prime} 2^{\prime}\right) \mp(2 \leftrightarrow 3) \\
& +\left[\mp G_{c}\left(3 \overline{2}^{+}\right) G_{c}\left(2 \overline{2} 2^{\prime} 3^{\prime}\right) G_{c}\left(\overline{1} 1^{\prime}\right)+(\overline{1} \leftrightarrow \overline{2})\right] \mp[(2 \leftrightarrow 3)] \\
& +G_{c}\left(23 \overline{2}^{+} 1^{\prime}\right)\left[G_{c}\left(\overline{2} \overline{1} 2^{\prime} 3^{\prime}\right)+G_{c}\left(\overline{2} 2^{\prime}\right) G_{c}\left(\overline{1} 3^{\prime}\right)\right. \\
& \left.\left.\mp G_{c}\left(\overline{2} 3^{\prime}\right) G_{c}\left(\overline{1} 2^{\prime}\right)\right]\right\} \\
& \left.\mp\left\{1^{\prime} \leftrightarrow 2^{\prime}\right\}+\left\{1^{\prime} \leftrightarrow 2^{\prime} \leftrightarrow 3^{\prime}\right\}\right\}
\end{aligned}
$$


Now we consider selected approximations by neglecting the four-particle correlation and selecting special sets of diagrams. First let us show how the known channel approximations appear which are the screened ladder, the maximally crossed diagrams and the ladder diagrams. Then we will consider the pair-pair correlations and their influence on these three channels.

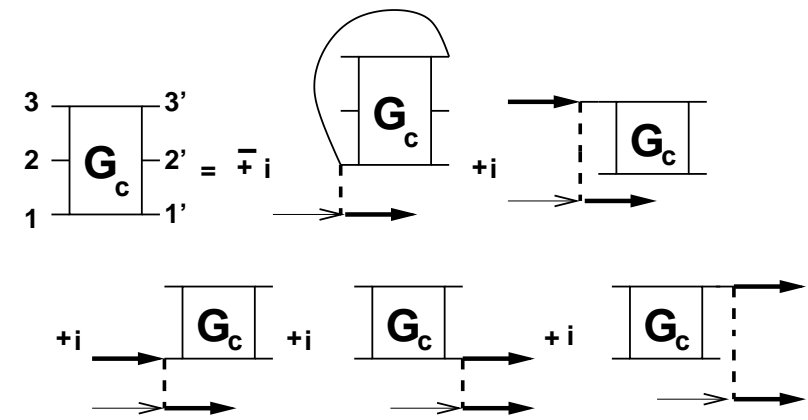

Fig. 5 A special set of diagrams for the three-particle correlated Green's function (25).

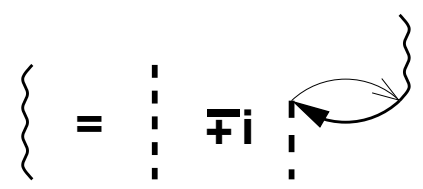

Fig. 6 Definition of the screened potential $V_{s}$.

\subsection{Screened ladder approximation}

We choose as partial summation from (25) the first set of diagrams indicated in figure 5. The diagrams obtained by interchanging $1^{\prime} \leftrightarrow 2^{\prime}$ and $1^{\prime} \leftrightarrow 2^{\prime} \leftrightarrow 3^{\prime}$ in figure 5 are contained in (25) as well. Now the iteration of the equation for the three-particle correlated Green's function in figure 5 leads to a repeated sum in the interaction lines which can be summarized by introducing the screened potential of figure 6. This procedure results into the expression for the three-particle correlated Green's function as illustrated in figure 7. Introducing this expression into the last diagram of figure 1 one obtains the diagrams of figure 8 . We see that the last line renormalizes the single-particle propagator if brought to the left-hand side. In such a way the equation of the two-particle Green's function in figure 8 can be very much simplified to 


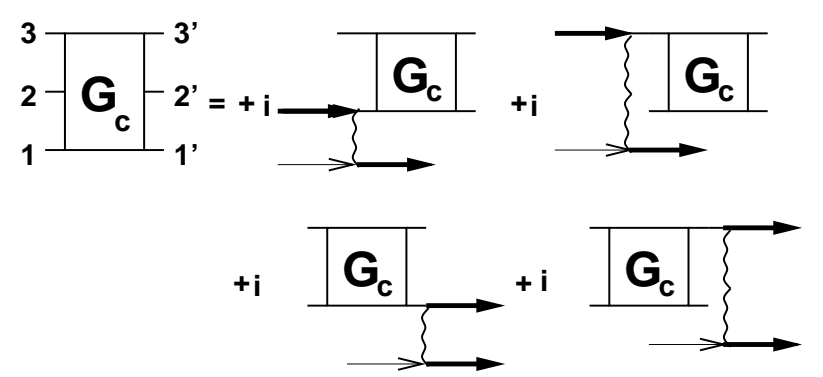

Fig. 7 The set of diagrams of figure 5 when introducing the screened potential of figure 6 .

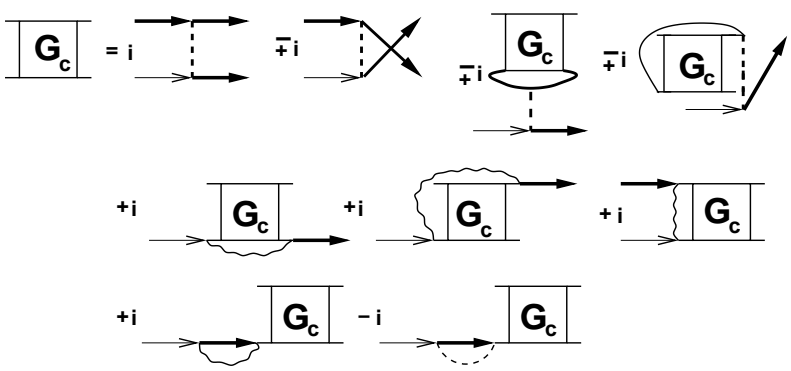

Fig. 8 The diagrams of figure 1 when introducing the three-particle Green's function of figure 7

which end we introduce the modified propagator

$$
\begin{aligned}
G_{s} & =G_{\mathrm{HF}}+G_{\mathrm{HF}}\left(\Sigma_{s}-\Sigma_{\mathrm{F}}\right) G_{s} \\
& =G_{\mathrm{H}}+G_{\mathrm{H}}\left(\Sigma_{s}\right) G_{s} \\
& =G_{0}+G_{0}\left(\Sigma_{s}+\Sigma_{\mathrm{H}}\right) G_{s}
\end{aligned}
$$

where $\Sigma_{s}(12)=i V_{s}(12) G(12)$ is the screened self energy and $\Sigma_{\mathrm{F}}(12)=$ $i V(12) G(12)$ the Fock self energy as illustrated in figure9. We will call in the

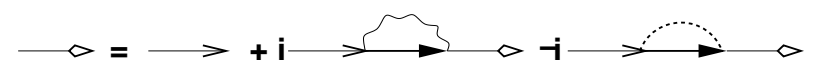

Fig. 9 The screened propagator (26).

following this modified propagator the channel-dressed selfconsistent propagator to distinguish him from the complete-dressed selfconsistent propagator. The channel-dressed propagator is determined by the corresponding selfenergy understood as the lowest selfconsistent diagram in the corresponding channel while the complete-dressed propagator includes all higher-order crossed terms. The introduction of this channel-dressed (screened) propagator results into the final expression of figure 10.

Compared to the Kadanoff-Martin approximation which was represented by diagrams of figure 1 neglecting the three-particle Green's function, we 


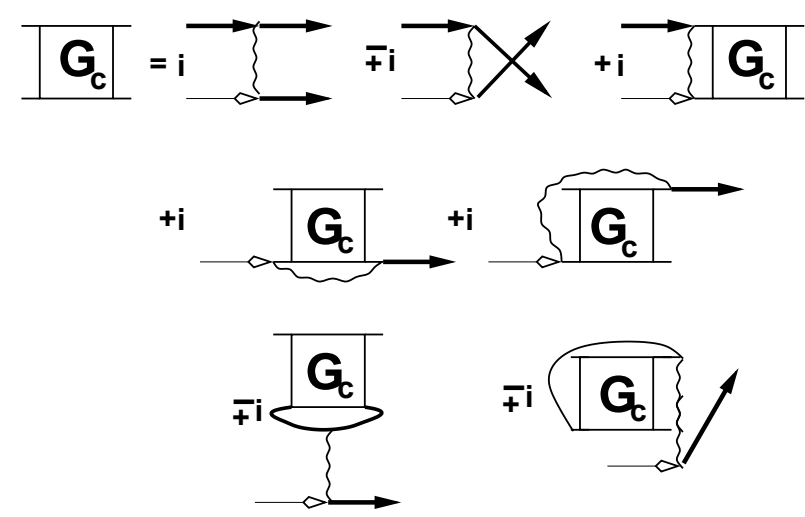

Fig. 10 The diagrams of figure 8 when introducing the channel-dressed propagator (26) indicated as thin open arrows.

now obtain in figure 10 the same set of diagrams except that the HartreeFock propagator has to be replaced by the modified one (26) and the bare interaction has to be replaced by the screened interaction of figure 6 in the ladders. Please note that the screened interaction appears with asymmetric propagators.

Again we see that for fermions and short range screened interaction the second and third line of diagrams in figure 10 are canceling mutually and only the screened ladder diagram remains. Together with the screened potential of figure 6] this is the screened ladder approximation used before [48] except that now one of the internal propagators has to be replaced by the screened one (26). This establishes the asymmetric form derived here as a new result.

Please note that (26) looks already like the structure of subtracting unphysical repeated collisions (24), however, only the Fock term appears to be subtracted from the screened approximation selfenergy in the propagator (26) since the scheme accounts for it already in the Bethe- Salpeter equation.

\subsection{Maximally crossed diagrams}

A next set of diagrams included in (25) is summarized by the maximally crossed ladders presented in figure 11 where the diagrams interchanging $1^{\prime} \leftrightarrow$ $2^{\prime}$ and $1^{\prime} \leftrightarrow 2^{\prime} \leftrightarrow 3^{\prime}$ are contained in (25) as well. We proceed with the same steps as in the last chapter by introducing the expression of figure 11 into the last diagram of figure 1 but defining now the channel-dressed propagator

$$
\begin{aligned}
G_{\tilde{T}} & =G_{0}+G_{0} \Sigma_{\tilde{T}} G_{\tilde{T}} \\
& =G_{\mathrm{HF}}+G_{\mathrm{HF}}\left(\Sigma_{\tilde{T}}-\Sigma_{\mathrm{HF}}\right) G_{T}
\end{aligned}
$$

to obtain the diagrams in figure 12. Here the selfenergy reads

$$
\Sigma_{\tilde{T}}\left(11^{\prime}\right)=\mp \int d \overline{1} \overline{2} \tilde{\Gamma}\left(\overline{1} 1 \overline{2} 1^{\prime}\right) G\left(\overline{2} \overline{1}^{+}\right)
$$




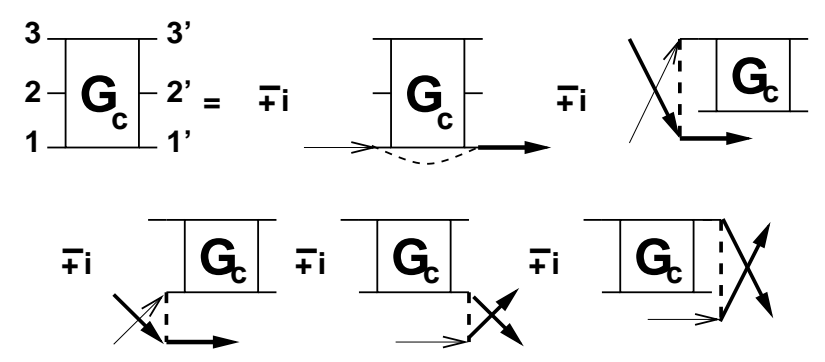

Fig. 11 The maximally crossed diagrams contained in (25).

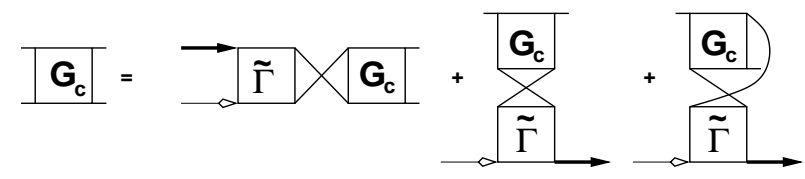

Fig. 12 The diagrams introducing figure 11 into figure 1 The thin open arrow mark now the channel-dressed propagator (27).

where the symmetrized vertex

$$
\tilde{\Gamma}(1234)=\tilde{T}(1234) \mp \tilde{T}(1243)
$$

is expressed via the maximally crossed ladders of figure 13 .

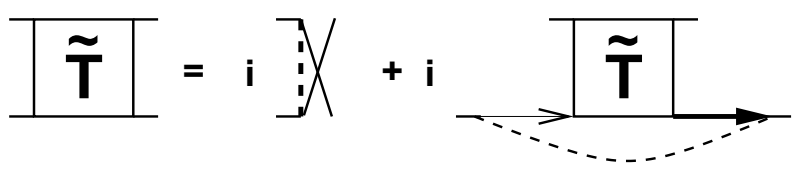

Fig. 13 The maximally crossed ladder summation.

\subsection{Ladder diagrams}

As a last set we select the ladder diagrams included in (25) which are collected in figure 14. Also the diagrams interchanging $2 \leftrightarrow 3$ are contained in (25).

Introducing the expression of figure 14 into the last diagram of figure 1 with the channel-dressed propagator

$$
\begin{aligned}
G_{T} & =G_{0}+G_{0} \Sigma_{T} G_{T} \\
& =G_{\mathrm{HF}}+G_{\mathrm{HF}}\left(\Sigma_{T}-\Sigma_{\mathrm{HF}}\right) G_{T}
\end{aligned}
$$

we obtain the diagrams in figure 15. Here the selfenergy reads

$$
\Sigma_{T}\left(11^{\prime}\right)=\int d \overline{1} \overline{2} \Gamma\left(\overline{1} 1 \overline{2} 1^{\prime}\right) G\left(\overline{2} \overline{1}^{+}\right)
$$

and the symmetrized vertex $\Gamma$ was given by (18) in terms of the ladder T-matrix (19) presented in figure 16. 


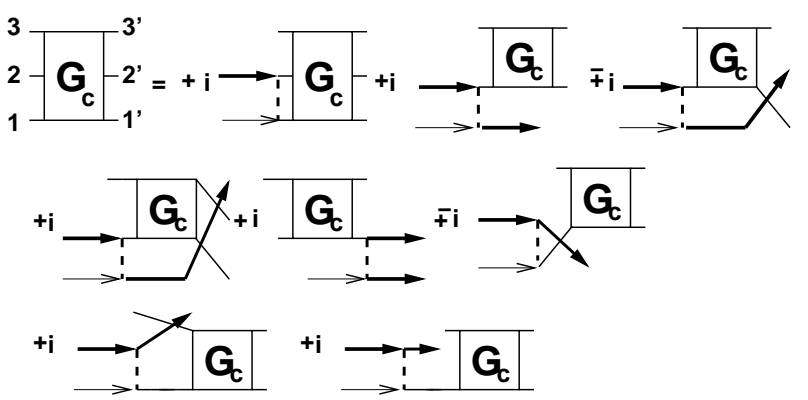

Fig. 14 The ladder diagrams contained in (25).

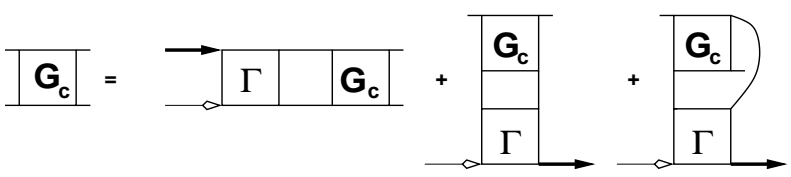

Fig. 15 The diagrams introducing figure 14 into figure 1 The thin open arrow are the channel-dressed propagator (30).

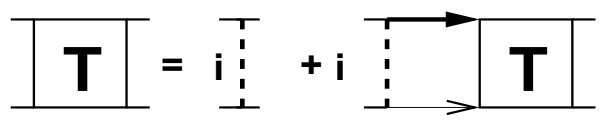

Fig. 16 The ladder summation.

\subsection{Pair-pair correlation}

Now that we have seen how the standard channels appear from the cummulant expansion of correlations in terms of Green's functions with special emphasis on the asymmetric propagators we proceed and investigate the pairpair correlation. In fact in (25) there are diagrams included which describe the interaction of two two-particle Green's functions outlined in figure 17.

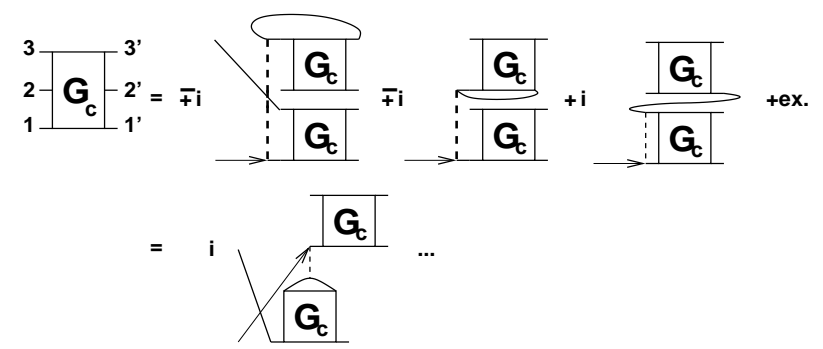

Fig. 17 The pair-pair diagrams in (25).

From these diagrams we search only the ones which yield a renormalization of the left-lower Hartree-Fock propagator after iteration in one of the above three channels, i.e. the first responsible diagram of figures 5 , 11 or 14 
respectively. In such a way we can define a channel-dressed propagator as done repeatedly above. It turns out that only the one diagram written in the second line of figure 17 fulfills this task. All other diagrams give repeated iterations partially included in the above summations and partially leading to new cross diagrams. These diagrams we will not consider here. They have been partially considered in Born approximation named as cluster HartreeFock diagrams 49 and have been applied to exciton problems 50 and to the first-order superfluid phase transition [51. These diagrams describe the interaction between the cluster and the single particle, while we concentrate here on a genuine cluster-cluster diagram.

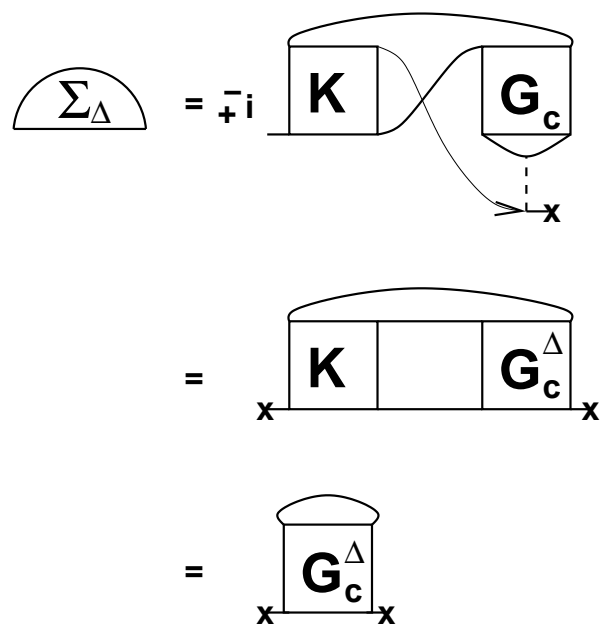

Fig. 18 The selfenergy of the renormalized propagator due to pair-pair correlations in figure 17 where the kernel $K$ signs $V_{s}-V$ for the screened channel of figure 10. $i \tilde{T}$ for the maximally crossed channel in figure 12 and $i T+V$ for the ladder diagrams in figure 15] The second line is valid only for separable two-particle correlations, see figure [19, when using the screened diagrams of figure 8 , The third line appears using the ladder diagrams of figure 15 Crosses denotes the inverse (amputated) propagator.

We introduce now this single renormalizing diagram of the last line in figure 17 into the corresponding three-particle ones on the right hand sides of figures 5, 11 or 14. This leads to iterations which sum the interactions and result into the channel effective blocks, screened potential, maximally crossed vertex or T-matrix vertex. These diagrams are then again introduced into the last diagram of figure 1 as done repeatedly before. In this way we obtain an additional renormalization diagram for the channel-dressed propagators of (26), (27) and (30) which can be written

$$
G_{\Delta}=G_{p}-G_{p} \Sigma_{\Delta} G_{\Delta}
$$

with $p=s, \tilde{T}, T$ denoting the channels. The corresponding selfenergy $\Sigma_{\Delta}$ due to the pair-pair interaction is shown in figure 18 where the kernel $K$ denotes the considered channels $V_{s}-V, i \tilde{T}$ and $i T+V$ correspondingly. 


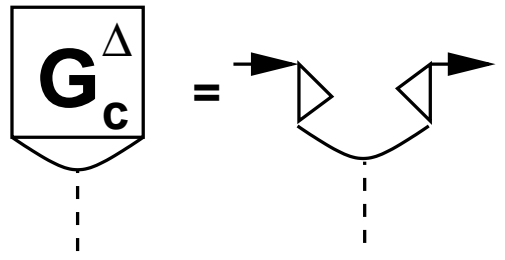

Fig. 19 Separation in in- and outgoing channels in the pole of pairing or condensation.

We want to consider specially the particle-particle channel represented by the T-matrix since in this channel the pairing appears. There we have

$$
\begin{aligned}
G_{\Delta}= & =G_{\mathrm{HF}}+G_{\mathrm{HF}}\left(\Sigma_{T}-\Sigma_{\Delta}-\Sigma_{\mathrm{HF}}\right) G_{\Delta} \\
& =G_{0}+G_{0}\left(\Sigma_{T}-\Sigma_{\Delta}\right) G_{\Delta} .
\end{aligned}
$$

For the singular channel, where the pairing appears, we will see now that the desired specific contribution remains. For this purpose we use the fact that the two-particle correlation separates near the pairing or condensation pole, illustrated in figure 19 and as it was used above in chapter 3.2. Taking this into account we see from the screened ladder diagrams in figure 8 that besides the Hartree and Fock terms which appear to be subtracted in figure 18. only the third term in figure 8 remains as a connected diagram. Therefore we can replace the corresponding form in the second part of figure 18 by a full two-particle propagator which results into the second line of figure 18 . Using the iteration of the T-matrix channel of figure 15 once more, we obtain the third line in figure 18 . Due to this procedure we obtain the result

$$
\Sigma_{\Delta}=\Sigma_{\chi}
$$

and comparing (24) with(33) we concluded that $G_{\Delta}=G_{x}$. This is exactly the subtracted propagator (24) proposed before which corrects for the repeated collisions with the same state. The derived selfenergy diagram in the first line of figure 18 is therefore one of the main results of this paper since it presents the diagrammatic part which leads to the subtraction of unphysical repeated collisions in any channel. For the particle-particle channel we have obtained the correct subtracted propagator and see that a proper collection of pair-pair correlations in the hierarchical expansion of correlations cares for the subtraction of such unphysical processes.

\section{Summary and outlook}

We have re-examined the Martin-Schwinger hierarchical structure of correlations. The expansion scheme of Green's functions has been written with the focus on the cummulant expansion form. We have investigated the binary collision approximation as well as three-particle correlations and have derived the corresponding Bethe-Salpeter equations. It appears that the two internal propagators of the resulting Bethe-Salpeter equations are asymmetric with 
respect to self-consistency. Dependent on the considered channel we obtain one complete-selfconsistent and one channel-dressed selfconsistent propagator. The binary collision approximation leads to one Hartree-Fock and one selfconsistent propagator in agreement with the Kadanoff and Martin form. This leads to the gap equation of pairing while the standard Bethe-Salpeter equation with two selfconsistent propagators does not.

We have proceeded to understand this asymmetry as a subtraction of repeated collision with the same state which is unphysical. Such single-channel corrections contribute to diagrammatic sums with a weight of the inverse volume and appears therefore only if one has a singular channel carrying a condensation with a macroscopic number of occupation. It is shown that such a subtraction scheme appears if we consider the correlations of two pairs of particles contained in the three-particle correlated Green's function. We identify the selfenergy diagram which is responsible for such proper subtraction scheme and show that the recently proposed scheme following the idea of Soven has its justification in the hierarchical dependence of correlations.

The here derived selfenergy correction responsible for this subtraction scheme is valid in all three considered channels, the screened ladder, the maximally crossed diagrams and the T-matrix channel. Though we had searched only for the correction of the latter one in order to obtain pairing and the gap equation we suggest that this selfenergy diagram might have an effect also to the maximally crossed diagrams. In that channel one describes weak localization phenomena. Since it was shown recently 43 that the parquet diagrammatic summation leads not to the expected Anderson localization, we suggest that the asymmetric parquet summation which appears due to the here derived selfenergy correction might solve these problems with localization. This investigation is deserved for further work.

Acknowledgements The discussions with Pavel Lipavský, Bretislav Šopík, Michael Männel and Alvaro Ferraz are gratefully mentioned. This work was supported by DFG-CNPq project 444BRA-113/57/0-1 and the DAAD-PPP program (BMBF). The financial support by the Brazilian Ministry of Science and Technology is acknowledged.

\section{A Variational technique, Ward identities and $\Phi$-derivability}

Due to the coupling of an external potential $U\left(11^{\prime}\right)=\delta_{11^{\prime}} U(1)$ one expresses the two-particle causal Green function $G\left(121^{\prime} 2^{\prime}\right)=1 / i^{2}<T \Psi_{1} \Psi_{2} \Psi_{2}^{+} \Psi_{1}^{+}>$by a variation of the one-particle Green's function $G(12)=1 / i<T \Psi_{1} \Psi_{2}^{+}>$with respect to the external potential [52,53] as

$$
G\left(121^{\prime} 2^{\prime}\right)=G\left(11^{\prime}\right) G\left(22^{\prime}\right) \mp \frac{\delta G\left(11^{\prime}\right)}{\delta U\left(2^{\prime} 2\right)}
$$

where the upper sign denotes the Fermi and the lower the Bose functions. Using the Dyson equation

$$
G^{-1}=G_{0}^{-1}-\Sigma-U
$$


one can calculated the derivative in (35) and with the help of the chain rule and $\delta G=-G \delta G^{-1} G$, the fluctuation function reads

$$
\begin{aligned}
L\left(121^{\prime} 2^{\prime}\right) & =G_{c}\left(121^{\prime} 2^{\prime}\right)+G\left(12^{\prime}\right) G\left(21^{\prime}\right) \\
& =G\left(121^{\prime} 2^{\prime}\right)-G\left(11^{\prime}\right) G\left(22^{\prime}\right) \\
& =\mp G\left(12^{\prime}\right) G\left(21^{\prime}\right) \mp G(13) \frac{\delta \Sigma(34)}{\delta U\left(2^{\prime} 2\right)} G\left(41^{\prime}\right) \\
& =\mp G\left(12^{\prime}\right) G\left(21^{\prime}\right)+G(13) \frac{\delta \Sigma(34)}{\delta G(56)} L\left(5262^{\prime}\right) G\left(41^{\prime}\right) .
\end{aligned}
$$

Double occurring indices are understood as integrated over. Equation (37) is expressed graphically in figure 20

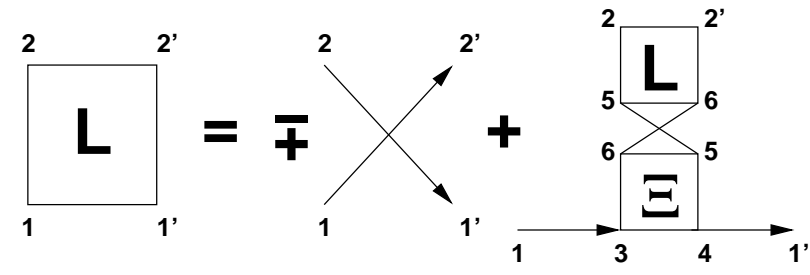

$$
{ }_{3}^{6}{ }_{4}^{5}=\frac{\delta \Sigma_{34}}{\delta G_{56}}=\Xi_{3645}
$$

Fig. 20 Variational representation of the two-particle correlation function (37)

Defining a three-point vertex function

$$
\begin{aligned}
\Gamma(123) & =\delta_{12} \delta_{13}+\frac{\delta \Sigma(12)}{\delta U(33)} \\
& =\delta_{12} \delta_{13}+\frac{\delta \Sigma(12)}{\delta G(45)} G(46) \Gamma(673) G(75)
\end{aligned}
$$

illustrated in figure 21 the two-particle correlation function of figure 20 can be represented by figure 22

The first line of equation (38) establishes the Ward identity [54,55, 56 which is therefore a consequence of the variational expression (35) and the form of Dyson equation (36). This Ward identity is also a consequence of the fact that the selfenergy can be represented as variation of a $\Phi$-functional with respect to the propagator

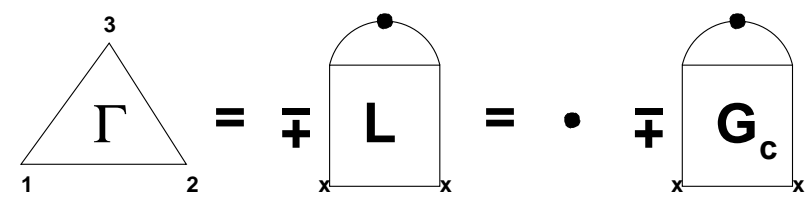

Fig. 21 Definition of the vertex function (38) 


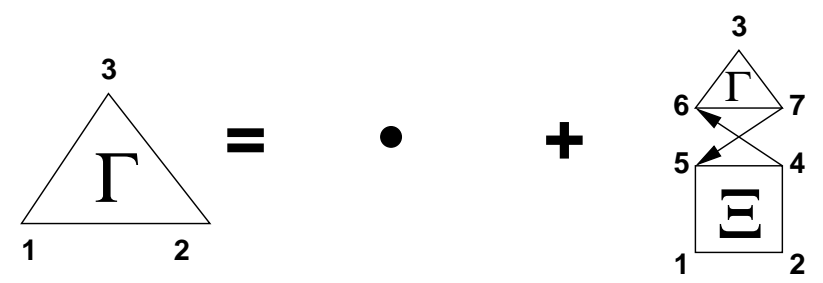

Fig. 22 Equation for the vertex function.
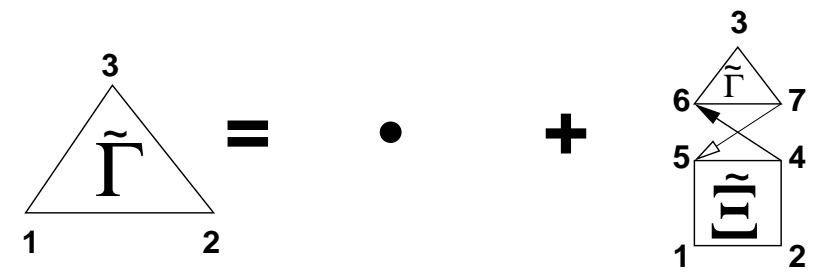

Fig. 23 Equation for the vertex function in asymmetric form.

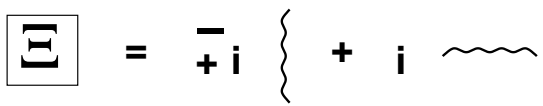

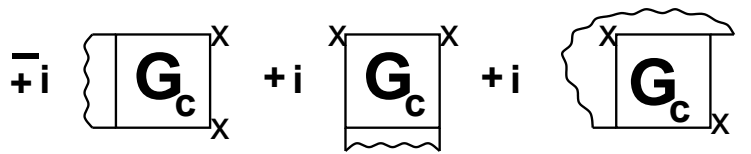

Fig. 24 Kernel of the vertex function in figure 22 for screened ladder channel of figure 10 .

39. Recently it has been proposed 57 to expand the variational scheme in terms of the kernel represented in the last line of figure 20.

How does this scheme fit now the asymmetric form presented in this paper? We demonstrate this on the example of the screened ladder approximation of figure 10. The other channels can be treated analogously. Closing the upper lines of the mirrored diagrams of figure 10 with the channel-corrected propagator we obtain the same form of the three-point vertex in figure 22 but now translated into figure 23 with asymmetric propagators. The kernel takes the form represented by figure 24 which can be verified by expanding the diagrams with the help of $G_{c}$ of figure 10. In comparison with figure 22 the intermediate propagators are asymmetric.

Using the Dyson equation for the channel-dressed propagator with channel index $i$ we can replace in one propagator of figure 22

$$
G=G_{i}+G_{i} \Sigma_{i} G
$$

and then have to show that

$$
\Xi(1234)+\Xi(1235) G_{i}(56) \Sigma_{i}(64) \equiv \tilde{\Xi}(1234)
$$

in order to proof $\tilde{\Gamma}=\Gamma$. To complete (40) we use $1+G \Sigma_{i}=G G_{i}^{-1}$ and write

$$
\begin{aligned}
\tilde{\Xi}(1234) & =\Xi(1235) G_{i}(56) G_{i}^{-1}(64) \\
& \equiv \frac{\delta \Sigma(12)}{\delta \mathcal{G}(43)}=\frac{\delta \Sigma(12)}{\delta G(56)} \frac{\delta G(56)}{\delta \mathcal{G}(43)}
\end{aligned}
$$


where we have introduced the (variational) selfconsistent propagator $\mathcal{G}$ to be defined by

$$
\frac{\delta G(56)}{\delta \mathcal{G}(43)} G_{i}(42) G(61) \equiv G(31) G(52)
$$

illustrated in figure 25.

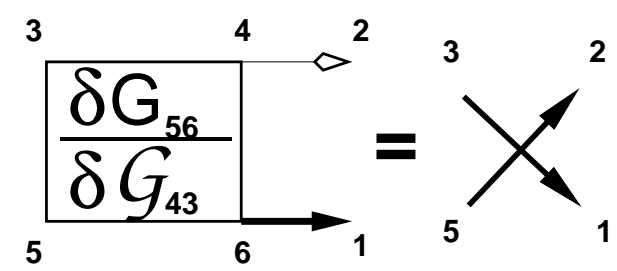

Fig. 25 Definition of the variational propagator $\mathcal{G}$ (42).

With the help of this variational propagator we can fulfill the relation (41) such that we have

$$
\tilde{\Xi}(1234)=\frac{\delta \Sigma(12)}{\delta \mathcal{G}(43)}
$$

This, however, has the required form of figure 20 such that (37) is fulfilled and the Ward identities are completed. Please note that neither $G$ nor $G_{i}$ serve as propagators to perform the variation but the variational propagator $\mathcal{G}$ defined by (42).

Therefore the screened ladder approximation (and the other 2 considered channels as well) can be recast into a form of three-point vertex function which obeys an integral equation with has the desired form of Ward identity. The difference to the traditional treatment is that a full dressed propagator is combined with the channel-dressed propagator. The price we pay is that we do have two different selfconsistent propagators the channel-dressed one and the complete dressed one.

Now we can write down the standard $\Phi$-functionals for T-matrix, screened ladder and maximally crossed channels but with symmetrical $\mathcal{G}$ propagators.

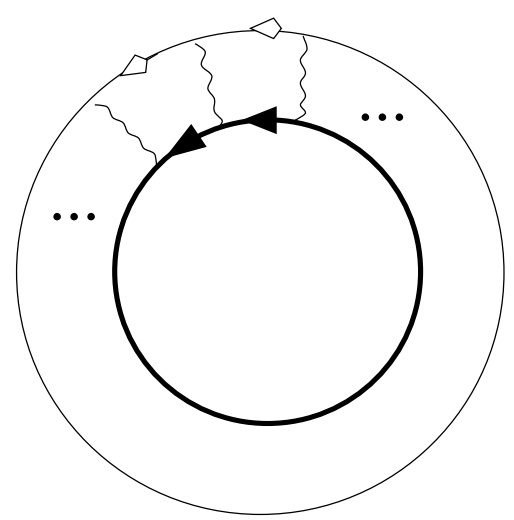

Fig. 26 The $\Phi$-functional defining the asymmetric screened channel approximation. 
Alternatively we can use (42) to replace them by the two different selfconsistent propagators. Consequently, the appearance of two different propagators does not violate the $\Phi$-derivability and the conservation laws. This can be considered alternatively by translating the known conserving approximations into forms of two different species in the system and interpret in the end one specie as the complete-dressed selfconsistent and the other specie as the channel-dressed selfconsistent propagator. For the screened ladder channel we declare e.g. a $\Phi$-functional as illustrated in figure 26 .

At the end it has to be remarked that the here proven Ward identity and $\Phi$ derivability is in a sense a triviality as long as the scheme is kept exact. Any formal expansion is 'exact' and will obbey therefore these exact identities. The difference between different expansion schemes lays in the different kind of approximations 'naturally' offered. Therefore at any stage of approximation actually used, one has to ensure the Ward identity. The introduction of the variational propagator (42) in figure 25 shows that any asymmetric approximation proposed with the scheme in this paper can be translated into a symmetric one. However the relation is very cumbersome in such a way that any asymmetric form chosen translates into many higher order diagrams in the symmetric writing.

\section{References}

1. L.P. Kadanoff, P.C. Martin, Phys. Rev. 124(3), 670 (1961)

2. R.F. Bishop, M.R. Strayer, J.M. Irvine, Phys. Rev. A 10(6), 2423 (1974)

3. R.F. Bishop, M.R. Strayer, J.M. Irvine, J. of Low Temp. Phys. 20(5-6), 573 $(1975)$

4. J. Maly, B. Jankó, K. Levin, Phys. Rev. B 59(2), 1354 (1999)

5. Y. He, C.C. Chien, Q. Chen, K. Levin, Phys. Rev. B 76(22), 224516 (2007)

6. C.C. Chien, Y. He, Q. Chen, K. Levin, Phys. Rev. A 77(1), 011601 (2008)

7. Q. Chen, J. Stajic, S. Tan, K. Levin, Phys. Rep. 412, 1 (2005)

8. W. Wild, Z. Phys. A 158(3), 322 (1960)

9. P. Lipavský, Phys. Rev. B 78, 214506 (2008)

10. K.M. Watson, Phys. Rev. 89, 575 (1953)

11. P. Soven, Phys. Rev. 156(3), 809 (1967)

12. K. Morawetz, Phys. Rev. B 81, 092501 (2010)

13. B.B. Kadomtsev, Zh.Eksp.Teor.Fiz. 33, 151 (1958). Sov. Phys. -JETP $33,117(1958)$

14. Y.L. Klimontovich, W. Ebeling, Jh. Eksp. Teor. Fiz. 63(3(9)), 904 (1972)

15. W. Ebeling, Ann. Phys. 33, 5 (1976)

16. W. Ebeling, G. Röpke, Ann. Phys. (Leipzig) 36, 429 (1979)

17. G. Röpke, Phys.Rev.A 38(6), 3001 (1988)

18. K. Morawetz, D. Kremp, Phys. Lett. A 173, 317 (1993)

19. A. Esser, G. Röpke, Phys. Rev. E. 58, 2446 (1998)

20. K. Morawetz, Phys. Rev. E 62, 6135 (2000). Errata 69, 029902

21. P. Debye, E. Hückel, Phys. Zeitsch. 15, 305 (1923)

22. L. Onsager, Phys. Zeitsch. 8, 277 (1927)

23. H. Falkenhagen, Elektrolyte (S. Hirzel Verlag, Leipzig, 1953)

24. H. Falkenhagen, W. Ebeling, W.D. Kraeft, in Ionic Interaction, ed. by Petrucci (Academic Press, New York and London, 1971), chap. 1, p. 1

25. D. Kremp, D. Kraeft, W. Ebeling, Ann. Phys. (Leipzig) 18, 246 (1966)

26. H.S. Köhler, R. Malfliet, Phys. Rev. C 48, 1034 (1993)

27. P. Bozek, Phys. Rev. C 59, 2619 (1999)

28. P. Bozek, Eur. Phys. J. A 15, 325 (2002)

29. W. Botermans, R. Malfliet, Phys.Rep. 198(3), 115 (1990)

30. P. Lipavský, K. Morawetz, V. Špička, Kinetic equation for strongly interacting dense Fermi systems, Annales de Physique, vol. 26,1 (EDP Sciences, Paris, 2001)

31. K. Bärwinkel, Z. Naturforsch. (a) 24, 38 (1969) 
32. H.B. Ghassib, R.F. Bishop, M.R. Strayer, Journal of Low Temperature Physics 23(3-4), 393 (1976)

33. Y. Noguchi, S. Ishii, K. Ohno, The Journal of Chemical Physics 125(11), $114108(2006)$

34. G. Toulouse, Phys. Rev. B 2(2), 270 (1970)

35. D.O. Gericke, S. Kosse, M. Schlanges, M. Bonitz, Phys. Rev. B 59(16), 10639 (1999)

36. R. Micnas, M.H. Pedersen, S. Schafroth, T. Schneider, J.J. Rodríguez-Núñez, H. Beck, Phys. Rev. B 52(22), 16223 (1995)

37. A. Vagov, H. Schomerus, A. Shanenko, Phys. Rev. B 76, 214513 (2007)

38. H. Puff, (Zentralinstitut für Elektronenphysik, Berlin, 1979)

39. P.C. Martin, J. Schwinger, Phys. Rev. 115, 1342 (1959)

40. P. Fulde, Electron Correlations in Molecules and Solids (Springer Series in Solid-State Sciences, Berlin, 1991)

41. G. Baym, L.P. Kadanoff, Phys. Rev. 124(2), 287 (1961)

42. V. Janis, Phys. Rev. B 64, 115115 (2001)

43. V. Janis, J. Phys: Condens. Matter (London) 21(48), 485501 (2009). DOI $\{10.1088 / 0953-8984 / 21 / 48 / 485501\}$

44. R.E. Prange, in Proc. of Int. Spring School of Physics, Naples (Academic Press of Japan, 1960)

45. B. Šopík, P. Lipavský, M. Männel, K. Morawetz, New J. Phys. (2010). In press

46. S.T. Beliaev, Soviet. Phys. JETP 7, 289 (1958)

47. L.P. Gorkov, Zh. Eksp. Fiz. 34, 735 (1958). Sov. Phys. JETP, 7 (1958) 505

48. R. Zimmermann, K. Kilimann, W. Kraeft, D. Kremp, G. Röpke, phys. stat. sol. (b) 90, 175 (1978)

49. G. Röpke, Ann. Physik (Leipzig) 506, 145 (1994)

50. G. Röpke, T. Seifert, H. Stolz, R. Zimmermann, phys. stat. sol. (b) 100, 215 (1980)

51. G. Röpke, Z. Phys. B 99, 83 (1995)

52. L.P. Kadanoff, G. Baym, Quantum Statistical Mechanics (Benjamin, New York, 1962)

53. W.D. Kraeft, D. Kremp, W. Ebeling, G. Röpke, Quantum Statistics of Charged Particle Systems (Akademie Verlag, Berlin, 1986)

54. J.C. Ward, Phys. Rev. 84(5), 897 (1951)

55. Y. Takahashi, Il Nuovo Cim. VI, 371 (1957)

56. B. Velický, A. Kalvová, V. Špička, Phys. Rev. B 77(4), 041201 (2008)

57. R. van Leeuwen, N.E. Dahlen, A. Stan, Phys. Rev. B 74(19), 195105 (2006) 